\title{
EDITORIAL
}

\section{Complementary Medicine and Pain Treatment: One Size does not Fit All}

\author{
"The physician of the future would not administer drugs, \\ but would involve the patient in the cure of human body structure \\ and functions, in alimentation, in disease causes and prevention".
}

Thomas Edison

In this special issue eminent researchers in the field review the use of some among the most used complementary therapies and techniques for the treatment of pain.

In the last decade, researchers have begun to understand better the complex interplay of neurons and messenger molecules that leads to the perception of pain. However, they are just now starting to explain how emotional affects and cognitive functions contribute to the experience of pain [1].

Since there are so many factors affecting pain perception and experience, the application of a traditional therapeutic model may fail as it does not consider the multidimensional nature of pain [2]. The keys to improving pain control are: good communication, comprehensive assessment of the patient, and thorough explanation of treatment options. Effective communication between doctor and patient can provide a more complete picture of the patient's pain experience. According to this perspective, complementary and alternative treatments are often incorporated into the care offered by multidisciplinary pain management teams, and include a wide range of techniques [3].

Voltaire stated that "doctors are men who prescribe medicines of which they know little, to cure diseases of which they know less, in human beings of whom they know nothing". Nowadays, doctors know almost everything about the drugs they prescribe, much about the diseases they cure, but still almost nothing about the ill person.

What Edison stated at the beginning of the last century is just what is practiced nowadays by the so-called holistic medicine, the medicine of the wholeness, considering human beings as an undivided whole of body-mind-spirit.

On this concept of unity all unconventional medicines are based. Frustration due to poor experience of pain management may lead patients to consult alternative practitioners as a last resort [4]. Alternative therapies can offer patients an individual approach tailored to their specific needs. Patients may perceive benefit from an empathetic approach and the feeling that their pain is taken seriously [5].

Actually, the principle critique against alternative therapies deals with their lack of scientific rigor. Evidence is crucial, but it needs to be based on solid scientific foundations [6]. Hippocrates used to say: "There are in fact two things, science and opinion; the former begets knowledge, the latter ignorance".

Placebo effect is often alleged by conventional medicine as an explanation of unconventional therapies results. Yet, it could be suspected that placebo effect is advocated to cover the discomfort that scientific minds experience when faced with the unusual [7].

Then, disciplines such as acupuncture and shiatsu, dating from 3,000 years, should wait for their scientificity to be proved before being applied? If complementary medicine's successes are dismissed because they are deemed impossible, the evidence will never be gathered, and medical knowledge may lose valuable opportunities to move into new areas.

After all, many medical advances begin with anecdote. It is interesting to remember the episode of Ignaz Semmelweis, an Austrian physician dealing with puerperal fever in Vienna at the end of the 19th century [8]. He found out that the problem was due to a hygienic negligence: operators used to make autopsies and then take deliveries without washing their hands. Therefore, he suggested to his colleagues that they washed the hands with soap after each intervention: an apparently banal, simple, practically costless action that modern doctors look at as absolutely mandatory. Well, doctors of those times raised such a war against their colleague that finally brought him to madhouse, and 50 years had to pass before recognizing he was right! How many people have died in those 50 years? How many sepses could have been avoided? It is clear how times for recognition and acceptance of a given reality or innovations by the conventional medicine are not as fast as one would expect.

Complementary medicine is still marginalized in the healthcare systems of many countries, but calls for a more integrated approach are growing louder and seem to be having an effect [9]. Hostility to complementary medicine among doctors is starting to erode. Basic courses in various complementary therapies are now available in many medical schools in the United States 
and Europe. Complementary therapies are usually used alongside conventional treatments, rather than as a substitute [10]. Pain syndromes (low back pain, joint pain, headache) are among the major presenting complaints of unconventional therapies.

The World Health Organization, the European Parliament and many countries have adopted or are in the process of adopting their own guidelines and coordination projects for the so-called "non conventional medicines". Global wellbeing, obtained non invasively, is winning more and more public favor and is including a growing number of interested people, which often are converted to the several unconventional practices available to date: from Chinese Medicine to acupuncture, from homeopathy to phytotherapy, from Ayurveda to manipulative techniques and Shiatsu.

Prevailing among all other therapies is undoubtedly homeopathy: the most used and integrated with other conventional therapies. The user is characterized by a middle-high school education. The tendency to use unconventional therapies increases with increasing qualification. Higher is qualification, greater is people self-determination. The typical consumer is about 30-40 years old. Women using unconventional therapies are more than men. Children are among the more frequent users of unconventional treatments. Among those who believe that unconventional therapies are useful, there is a common tendency to ascribe to them a lower toxicity than conventional therapies.

Most complementary techniques are defined in terms of a historical tradition and discussing their recent advances may seem contradictory [11]. Yet, substantive changes in the scientific base and organizational structure of complementary medicine have occurred recently, indicating that it is becoming more integrated. Integration has obvious implications for: 1) access to and availability of care; 2) practitioners' agreement on their respective roles; 3) patients feeling they are receiving care as part of a coordinated service [12].

Complementary therapies represent valuable strategies, particularly for the treatment of acute and chronic pain. If the principles of unconventional therapies could be combined with the efficacy of an appropriate pharmacological regimen, as well as with other conventional techniques, much of the pain could be avoided. Perhaps now is the time to recognize the limitations of the "one size fits all policy" and to embrace a multidimensional approach to pain management [13].

Each of us is free to believe or not to unconventional medicine, according to his/her own knowledge, experience, sensations. But what none of us should ever forget is to always keep an open mind! "The mind is like a parachute, it only works when it is open."

\section{REFERENCES}

[1] Wiech K, Ploner M, Tracey I. Neurocognitive aspects of pain perception. Trends Cogn Sci 2008; 12: 306-13.

[2] Patel G, Euler D, Audette JF. Complementary and alternative medicine for noncancer pain. Med Clin North Am 2007; 91: 141-67.

[3] Haetzman M, Elliott A, Smith B, et al. Chronic pain and the use of conventional and alternative therapy. Fam Pract 2003; 20: 147-54.

[4] Paterson C, Britten N. 'Doctors can't help much': the search for an alternative. Br J Gen Pract 1999; 49 : 626-9.

[5] Austin JA. Why patients use alternative medicine. JAMA 1998; 279: 1548-53.

[6] Ernst E. Complementary and alternative medicine: between evidence and absurdity. Perspect Biol Med 2009; 52(2): 289-303.

[7] Di Blasi Z, Kleijnen J. Context effects. Powerful therapies or methodological bias? Eval Health Prof 2003; 26: 166-79.

[8] Noakes TD, Borresen J, Hew-Butler T, et al. Semmelweis and the aetiology of puerperal sepsis 160 years on: an historical review. Epidemiol Infect 2008;136: 1-9.

[9] Ernst E. Prevalence of use of complementary/alternative medicine: a systematic review. Bull World Health Organ 2000; 78 : 252-7.

[10] Ernst E. The role of complementary and alternative medicine. BMJ 2000; 321: 1133-35.

[11] Carter B. Methodological issues and complementary therapies: researching intangibles? Complement Ther Nurs Midwifery 2003; 9: 133-9.

[12] Carr A, George DS, Read N, et al. Integrated medicine. BMJ 2001; 322: 1484.

[13] Baldwin E. Time for a fresh look at complementary medicine. BMJ 2003; 326: 1322.

Marco Luchetti (Guest Editor)

The Open Pain Journal Department of Anesthesia Intensive Care \& Pain Management "A. Manzoni" General Hospital Via dell'Eremo 9/11, 23900 Lecco Italy Tel: +390341489984 Fax: +390341489983

E-mail:m.luchetti@fastwebnet.it m.luchetti@ospedale.lecco.it 